\title{
Evolution of structure and local magnetic fields during crystallization of HITPERM glassy alloys studied by in situ diffraction and nuclear forward scattering of synchrotron radiation
}

\author{
Marcel Miglierini ${ }^{1,2}$, Márius Pavlovič ${ }^{1}$, Vít Procházka ${ }^{3}$, Tomáš Hatala ${ }^{1 *}$, Gerhard \\ Schumacher ${ }^{4}$, and Rudolf Rüffer ${ }^{5}$ \\ ${ }^{1}$ Institute of Nuclear and Physical Engineering, Slovak University of Technology, \\ Ilkovičova 3, 81219 Bratislava, Slovakia \\ ${ }^{2}$ Regional Centre of Advanced Technologies and Materials, Palacky University, \\ 17. listopadu 12, 77146 Olomouc, Czech Republic \\ ${ }^{3}$ Department of Experimental Physics, Faculty of Science, Palacky University, \\ 17. listopadu 12, 77146 Olomouc, Czech Republic \\ ${ }^{4}$ Helmholtz-Zentrum, Berlin für Materialien und Energie GmbH, Hahn-Meitner Platz 1, \\ D-14109 Berlin, Germany \\ ${ }^{5}$ ESRF-The European Synchrotron, CS40220, 38043 Grenoble Cedex 9, France
}

\begin{abstract}
Evolution of structure and local magnetic fields in $\left(\mathrm{Fe}_{1-\mathrm{x}} \mathrm{Co}_{\mathrm{x}}\right)_{76} \mathrm{Mo}_{8} \mathrm{Cu}_{1} \mathrm{~B}_{15}$ (HITPERM) metallic glass ribbons with various amounts of Co $(x=0,0.25,0.5)$ were studied in situ using diffraction and nuclear forward scattering of synchrotron radiation. It was found that crystallization for all three glasses proceeds in two stages. In the first stage, bcc (Fe,Co) nanocrystals are formed, while in the second stage additional crystalline phases evolve. For all three glasses, the crystallization temperatures at the wheel side were found to be lower than at the air side of the ribbon. The crystallization temperatures were found to decrease with increasing Co content. The lattice parameters of the bcc nanocrystals decrease up to about $550{ }^{\circ} \mathrm{C}$ and then increase pointing to squeezing Mo atoms out of the nanograins or to interface effects between the nanocrystals and the glassy matrix. Nuclear forward scattering enabled separate evaluation of the contributions that stem from structurally different regions within the investigated samples including the newly formed nanocrystals and the residual amorphous matrix. Even minor Co content $(x=0.25)$ has a substantial effect not only upon the magnetic behaviour of the alloy but also upon its structure. Making use of hyperfine magnetic fields, it was possible to unveil structurally diverse positions of $\mathrm{Fe}$ atoms that reside in a nanocrystalline lattice with different number of Co nearest neighbours.
\end{abstract}

\section{Keywords:}

Nanocrystalline alloys, chemical substitution, diffraction of synchrotron radiation, nuclear forward scattering of synchrotron radiation, structural transformations, hyperfine interactions.

\footnotetext{
*) Current address: Vacuumschmelze, s.r.o, Horná Streda 1325/14, 91624 Horná Streda, Slovakia
} 


\section{Introduction}

Nanocrystalline metallic alloys represent a new class of materials with unique physical properties that are suitable for variety of practical applications [1]. There are three families of nanocrystalline alloys (NCAs), namely FINEMET [2], NANOPERM [3], and HITPERM [4]. They all can be prepared from metallic glasses (MGs) with suitable chemical composition that are annealed under well defined conditions (temperature and time). Annealing induces partial crystallization that is characterized by formation of crystalline grains with typical sizes of up to several tens of nanometres. Physical properties of the NCAs can be tailored not only with the aid of their chemical composition, but also by varying the size of the nanocrystalline grains, their morphology, and composition. Thus, structural transformations obtained through crystallization considerably affect macroscopic physical properties of these materials.

Crystallization is a one-way process of phase transformation that governs the structural order. In order to understand macroscopic properties of the NCAs, it is inevitable to understand their microstructure as well as physical consequences of structural transformations. The latter are accompanied by changes in hyperfine interactions that, consequently, modify the overall magnetic properties. In contrary to MGs, the magnetic parameters of the NCAs do not substantially deteriorate at elevated temperatures [5, 6], which might occur at some practical applications.

In order to understand the correlation between structural arrangement and macroscopic magnetic properties, both amorphous MGs and NCAs are investigated by a broad arsenal of diagnostic techniques. In our earlier works, we used differential scanning calorimetry (DSC), $\mathrm{X}$-ray diffraction, transmission electron microscopy, high resolution electron microscopy, electron diffraction, scanning microscopy, Mössbauer spectrometry, nuclear magnetic resonance spectroscopy, atomic field microscopy and conventional magnetic measurements [7-12]. Nevertheless, the majority of these techniques can provide only ex situ information as the time needed for acquisition of sufficiently good statistics of the experimental data frequently extends over several tens of minutes or even hours. Subsequently, in situ investigation of the induced structural transformations is not possible in real time using these techniques.

There are few methods suitable for in situ investigations. However, they usually scan the whole bulk of the investigated systems and provide information that is averaged over all structurally different regions. These comprise for example DSC and/or magnetic measurements. Thus, the in situ characterization of structural transformations during crystallization of MGs is an ambitious task. This is especially true when information on an atomic level is of interest. To ensure this, advanced in situ characterization analytical tools such as those using superior intense $\mathrm{X}$-ray beams produced by the third generation of the synchrotron radiation sources should be employed.

Formation of ultrafine microstructure in a FINEMET-type alloy was followed by Köster et al. [13] using in situ time resolved diffraction of synchrotron radiation (DSR). Their findings emphasised the role of diffusion of impurities during the transformation. Kinetics of crystallization of FINEMET-type MGs $[14,15]$ and the effect of Co upon the kinetics of crystallization [16] were also studied using in situ DSR. Structural relaxation processes in MGs studied by in situ DSR showed that microscopic structural changes can be correlated to macroscopic characteristics such as thermal expansion coefficient [17]. Furthermore, Poulsen et al. [18] demonstrated that deformation tensor components can be extracted from MGs using high quality synchrotron diffraction data. As far as bulk MGs are concerned, differences in their glass-forming abilities were pointed out to be responsible for different icosahedral arrangements in short-range order [19].

High Energy X-ray Diffraction (HEXRD) of synchrotron radiation was employed in situ 
to investigate the change of free volumes upon heating [20]. It was also engaged to measure plastic deformation mechanisms in MGs [21]. Recently, we have used in situ HEXRD (energy $\sim 60 \mathrm{keV}$ ) for the study of structural arrangements and relaxation processes induced in FINEMET-type MGs by swift heavy-ion bombardment [22]. Kinetics of crystallization was reported earlier in similar alloys using low energy ( $7 \mathrm{keV})$ in situ DSR [23].

As mentioned above, mostly FINEMET-type alloys have been studied by advanced in situ tools so far. In this work, we demonstrate the use of novel in situ characterization techniques based on synchrotron radiation in the investigation of (Fe,Co)-Mo-Cu-B nanocrystalline alloys with varying chemical composition. We have chosen this MoHITPERM system because of its interesting magnetic behaviour and also because it was thoroughly studied using conventional methods. Structural transformation, compositional dependence of Curie temperature, and magnetic behaviour of $\left(\mathrm{Fe}_{x} \mathrm{Co}_{1}\right)_{79} \mathrm{Mo}_{8} \mathrm{Cu}_{1} \mathrm{~B}_{12}(x=1,2$, 3, 6, 9, 12) and $\mathrm{Fe}_{79} \mathrm{Mo}_{8} \mathrm{Cu}_{1} \mathrm{~B}_{12}$ MGs were investigated by Conde et al. [24, 25]. Recently, changes in the magnetic properties provoked by the microstructural evolution were followed also by Mössbauer spectrometry [26]. Namely the latter technique enables mutual correlation between structural arrangement and magnetic order. Mössbauer spectrometry was extensively employed to examine hyperfine interactions of the as-quenched and annealed aforementioned MG as well as of the one with the same Fe/Co ratio but with higher amount of boron, that is $\left(\mathrm{Fe}_{1-x} \mathrm{Co}_{1}\right)_{76} \mathrm{Mo}_{8} \mathrm{Cu}_{1} \mathrm{~B}_{15}[27,28]$. Though both compositions behave very alike especially for higher Co contents, their original Co-free alloys are remarkably different from the magnetic order point of view. While the alloy with 12 at. \% of boron is paramagnetic at room temperature, weak magnetic interactions are observed for 15 at. \% of boron. That is why the latter system is better suited for the study of hyperfine magnetic interactions in the vicinity and above room temperature.

In this work, formation of nanocrystalline grains during structural transformation of a heat-treated original metallic glass is followed by in situ diffraction of synchrotron radiation. Extended information about changes in their magnetic states is obtained by nuclear forward scattering (NFS) of synchrotron radiation [29]. This method enables differentiation of the signal given by the amorphous residual matrix from the signal given by the newly formed nanocrystals. Because the experimental data are collected during very short time (about one minute), on-fly inspection of both structural and magnetic aspects of the transformation is possible. Furthermore, NFS can distinguish between atoms positioned in the bulk and those on the surfaces of the nanograins.

\section{Experimental Details}

\subsection{Investigated materials}

NANOPERM-type nanocrystalline alloys based on Fe-M-B-Cu, where $\mathrm{M}=\mathrm{Zr}$, Nb, Hf, Mo, ... have been proposed by Suzuki [3, 30]. These alloys show small magnetostrictive coefficients and, simultaneously large permeability. Substitution of cobalt atoms at the expense of iron in the (Fe,Co)-M-Cu-B -type alloys called HITPERM [4] shows high permeability and high Curie temperatures. Cobalt with its soft magnetic properties is important for set of magnetic properties in the final alloys.

As-quenched $\mathrm{MG}$ alloys $\left(\mathrm{Fe}_{1-\mathrm{x}} \mathrm{Co}_{\mathrm{x}}\right)_{76} \mathrm{Mo}_{8} \mathrm{Cu}_{1} \mathrm{~B}_{15}$ with various amount of $\mathrm{Co}(x=0,0.25$, 0.5 ) were prepared by the method of planar-flow casting on a rotating quenching wheel in a form of ribbons about $10 \mathrm{~mm}$ wide and $20 \mu \mathrm{m}$ thick. In addition, about 1-2 mm wide ribbons that were enriched to about $50 \%$ in the ${ }^{57} \mathrm{Fe}$ isotope were prepared for $x=0$ and 0.25 . Because the content of the ${ }^{57} \mathrm{Fe}$ in natural iron is only $\sim 2 \%$, these samples were used to 
facilitate the NFS experiments, i.e., to increase the count rate and so to shorten the acquisition time.

The side of the ribbons that was in direct contact with the quenching wheel will be referred to as the wheel side. The opposite side, i.e. the one exposed to the surrounding atmosphere during the production process will be called the air side. Chemical composition of the alloys enriched in ${ }^{57} \mathrm{Fe}$ was checked by optical emission spectrometry with inductively coupled plasma (Mo, B) and flame atomic absorption spectrometry (Fe, Co, Cu).

\subsection{Methods}

\subsubsection{Synchrotron radiation and its diffraction}

Synchrotrons represent unique sources of radiation that is characterised by outstanding properties [31]. The synchrotron radiation features tuneable energy, high degree of polarization, small beam cross-section, negligible beam divergence, pulsed time structure, and above all extremely high brilliance (intensity).

Tremendous brilliance of synchrotron radiation enables such diffraction experiments that are not feasible with conventional X-ray sources. For example, it is possible to acquire diffractograms during continuous temperature increase of a heat treated investigated sample and thus to observe in situ the process of structural transformation.

DSR was performed at the KMC-2 experimental station at BESSY II, Berlin with the energy of $7 \mathrm{keV}(\lambda=0.177121 \mathrm{~nm})$ and a photon flux of $\sim 2 \times 10^{10}$ photons/s. The estimated heat load at the sample was $0.1 \mathrm{~mW}$. The main (110) reflection maximum of a bcc-Fe lattice was registered using a 2-D Bruker HI-STAR detector. The data were acquired every $10 \mathrm{sec}$ with an angular resolution of $0.03^{\circ}$ in a standard $2 \theta$ scattering geometry. Using grazing incidence arrangement, the penetration depth of the radiation is $\sim 5 \mu \mathrm{m}$. In this way, surface studies are possible and that is why the diffractograms were recorded from both sides of the ribbon-shaped samples.

About $2 \mathrm{~cm}$ long pieces of as-quenched alloys were positioned in an evacuated hemispherical dome made from beryllium. The samples were attached to a heated holder and exposed to continuous heating with a temperature increase of $10 \mathrm{~K} / \mathrm{min}$ up to $800{ }^{\circ} \mathrm{C}$.

\subsubsection{Nuclear forward scattering of synchrotron radiation (NFS)}

Mössbauer spectroscopy (MS) is a method that directly correlates magnetic states of the studied materials with their structure through hyperfine interactions [7-10, 23]. It is, however, quite time consuming and thus not suitable for observation of rapid dynamic processes. With the availability of the third generation synchrotron sources, the method of nuclear forward scattering (NFS) of synchrotron radiation [32] became feasible. NFS makes use of the ${ }^{57} \mathrm{Fe}$ resonant atoms as probes of the local magnetic and electronic properties in the investigated samples and provide information on hyperfine interactions similarly to MS. Application of this technique is helpful in revealing the mutual relation between the magnetic arrangement and the structure of the studied materials and it can be used in dynamic in situ regimes.

The pulsed time structure of synchrotron radiation opens new horizons for experiments in the time domain. The synchrotron radiation beam is tuned to the energy of the requested Mössbauer isotope and highly monochromatized with an energy bandpass of meV. The beam impinges on to the sample, where all energetic levels of every Mössbauer nucleus present in the sample are excited at the same time during the short pulse of the synchrotron radiation. In the time slot between two subsequent pulses, all excited nuclei coherently emit the excess 
energy in form of resonance delayed photons that are registered with the fast detector.

The nuclear levels are often split due to hyperfine interactions as shown at the left-hand side of Fig. 1. Here, an example of magnetically split nuclear levels of ${ }^{57} \mathrm{Fe}$ is shown. Energy separation among the levels is of the order of $\mu \mathrm{eV}$. Thus, the synchrotron radiation with a bandwidth of several meV excites all nuclear transitions simultaneously. The successive decay is characterized by an interference of the nuclear transitions from all excited levels giving rise to the delayed signal in the time domain, viz. NFS interferogram. The latter exhibits characteristic beating of intensities called quantum beats. An example of such a record is shown on the right-hand side of Fig. 1.
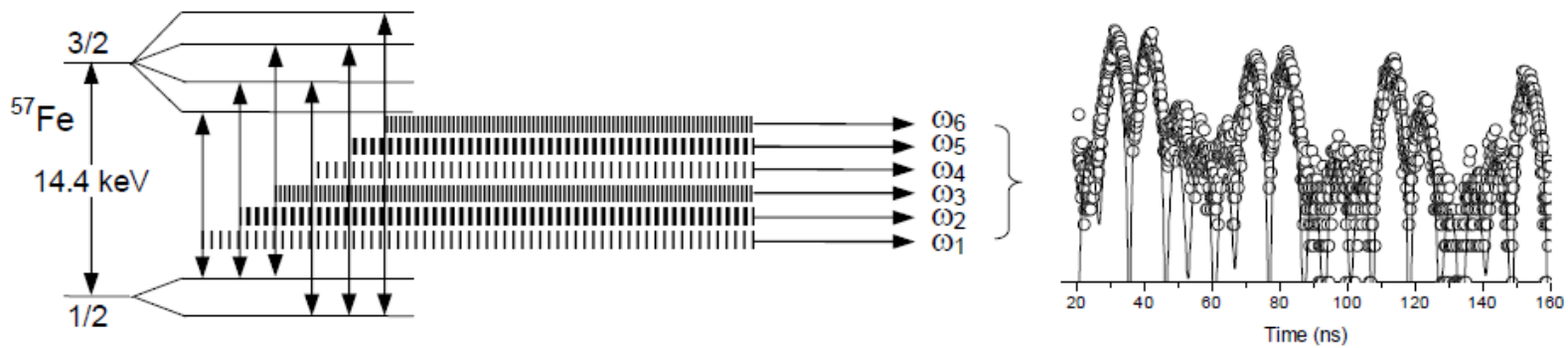

Fig. 1. Magnetically split nuclear levels of ${ }^{57} \mathrm{Fe}$ (left), photons from de-excitation transitions decays that are characterized by the frequencies (energies) $\omega_{1}$ to $\omega_{6}$ (middle), and the resulting interferogram (right).

The resulting NFS interferogram of the characteristic quantum beats carries information on electric quadrupole and magnetic dipole hyperfine interactions that are unique for individual atomic sites of the resonant atoms. The counts of delayed photons are registered as a function of the time that has elapsed after the excitation. That is why NFS is sometimes referred to as Mössbauer spectrometry in the time domain. Several applications of this technique to different problems of condensed matter physics including NFS in glassy and nanoscale materials are reviewed in [33].

NFS experiments were performed at the Nuclear Resonance side-station ID22N of the European Synchrotron Radiation Facility (ESRF), Grenoble. Excitation of the ${ }^{57} \mathrm{Fe}$ nuclear levels was accomplished by a photon beam with $14.413 \mathrm{keV}$ energy, $\sim 10^{9}$ photons/s flux, and $\sim 3 \mathrm{meV}$ bandwidth. The estimated heat load at the sample was $0.2 \mathrm{~mW}$. Samples were placed in a vacuum furnace and heated up with a ramping rate of $10 \mathrm{~K} / \mathrm{min}$ up to $670{ }^{\circ} \mathrm{C}$. The maximum annealing temperature was limited by Kapton windows of the furnace. The total time of annealing time was 65 minutes. The NFS interferograms were continuously recorded every minute during the whole annealing process in transmission geometry. Thus, information for the bulk of the sample is obtained. Samples enriched in the stable ${ }^{57} \mathrm{Fe}$ isotope were used in order to enable faster data acquisition. They were $\sim 1-2 \mathrm{~mm}$ wide while the spot-size of the synchrotron beam was of $0.7 \times 0.3 \mathrm{~mm}^{2}$. Evaluation of the experimental data was accomplished by the CONUSS software package [34, 35].

\section{Results and Discussion}

\subsection{Diffraction of synchrotron radiation}

The process of structural transformation of originally amorphous metallic glasses was followed by DSR taken from both sides of the ribbon-shaped samples. In order to speed-up the acquisition of experimental data, the position of the 2-D detector was fixed. Taking into 
consideration the distance between the sample and the detector as well as the sensitive area of the latter, it was possible to acquire the scattered signal from about $14^{\circ}$ of $2 \theta$ angles. That was enough for following the time evolution of the principal (110) reflection belonging to an expected bcc-Fe crystalline phase.

Diffractograms obtained for the $\left(\mathrm{Fe}_{1-x} \mathrm{Co}_{x}\right)_{76} \mathrm{Mo}_{8} \mathrm{Cu}_{1} \mathrm{~B}_{15}$ alloys are presented as contour plots in Figs. 2, 3, and 4 for $x=0,0.25$, and 0.5 , respectively. Vertical scales show the temperature of the sample and the horizontal ones represent $2 \theta$ angles from $48^{\circ}$ to $62^{\circ}$. This range was covered by the fixed 2-D detector and coincides with the position of the main (110) reflection at $\sim 52^{\circ}$. Intensities of the diffracted lines are colour coded as shown by the corresponding legends. The depicted temperatures $T$ extend from $300{ }^{\circ} \mathrm{C}$ up to $700{ }^{\circ} \mathrm{C}$ where the structural transformations of interest take place within this temperature interval. The temperature of the onset of the first and the second crystallizations $T_{x 1}$ and $T_{x 2}$, respectively, are marked in the figures with white lines.

(a)

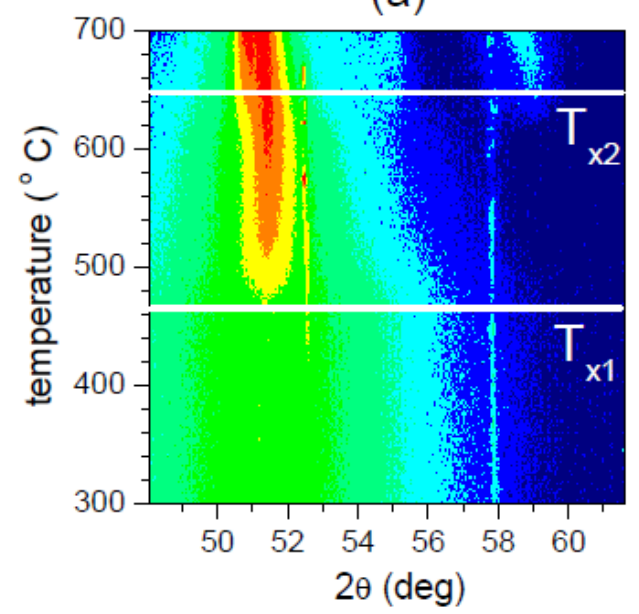

(b)

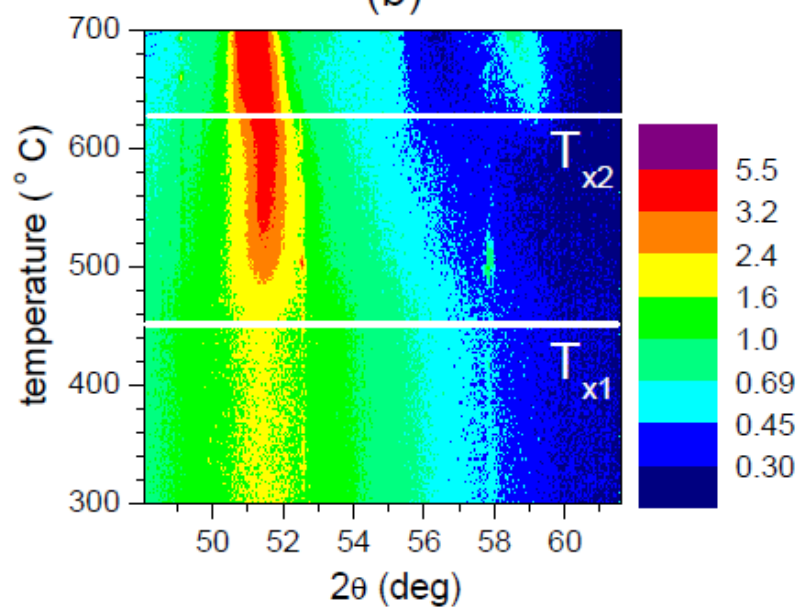

Fig. 2. Contour plots of diffractograms recorded by in situ DSR from the air (a) and the wheel (b) side of the $\left(\mathrm{Fe}_{1-x} \mathrm{Co}_{x}\right)_{76} \mathrm{Mo}_{8} \mathrm{Cu}_{1} \mathrm{~B}_{15}(x=0)$ alloy. Crystallization temperatures $T_{x 1}$ and $T_{x 2}$ are indicated by the horizontal white lines.

(a)

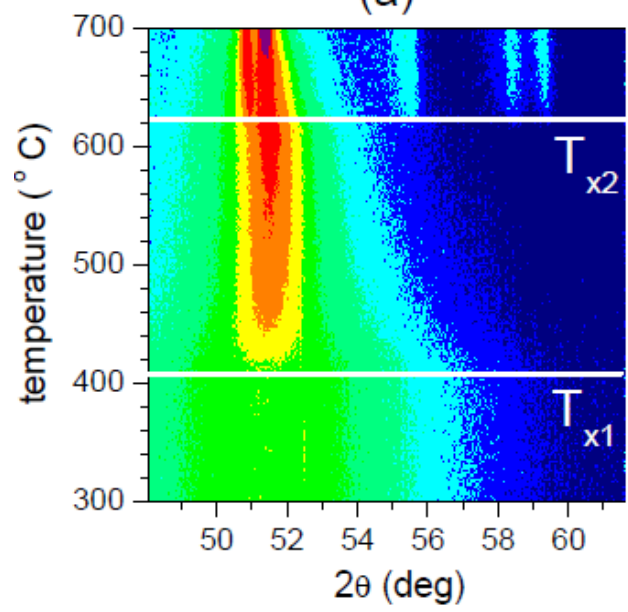

(b)

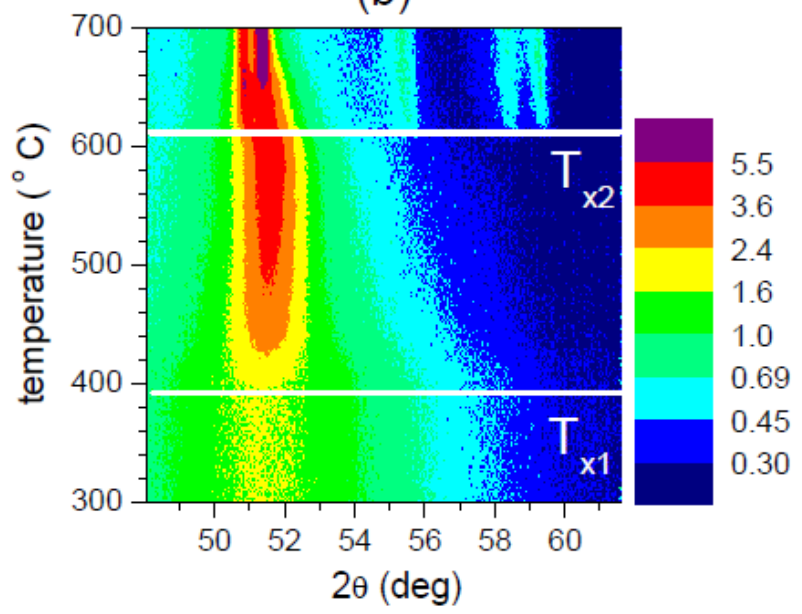

Fig. 3. Contour plots of diffractograms recorded by in situ DSR from the air (a) and the wheel (b) side of the $\left(\mathrm{Fe}_{1-x} \mathrm{Co}_{x}\right)_{76} \mathrm{Mo}_{8} \mathrm{Cu}_{1} \mathrm{~B}_{15}(x=0.25)$ alloy. Crystallization temperatures $T_{x 1}$ and $T_{x 2}$ are indicated by the horizontal white lines. 
The contour plots in Figs. 2-4 can be subdivided into three temperature regions. In the first region where $T<T_{x 1}$, the investigated alloys are fully amorphous. The corresponding diffractograms exhibit broad reflections that are spread over wide range of $2 \theta$ angles. The second temperature region with $T_{x 1}<T<T_{x 2}$ is characterized by the onset and formation of the first crystallization phase. This is identified by a single narrow reflection peak that progressively rises in intensity with annealing temperature. At the same time, a shift of the position of its maximum towards smaller $2 \theta$-values is observed. This can be ascribed to a lattice expansion with increasing temperature of the sample. For $x=0$ in Fig. 2, this crystalline phase belongs to bcc-Fe that is supersaturated with some Mo. The newly formed nanocrystals are only about 5-8 nm in size [11]. In the $x=0.25$ (Fig. 3) and $x=0.5$ (Fig. 4) alloys, the main diffraction peak corresponds to bcc-(Fe,Co) phase.

(a)

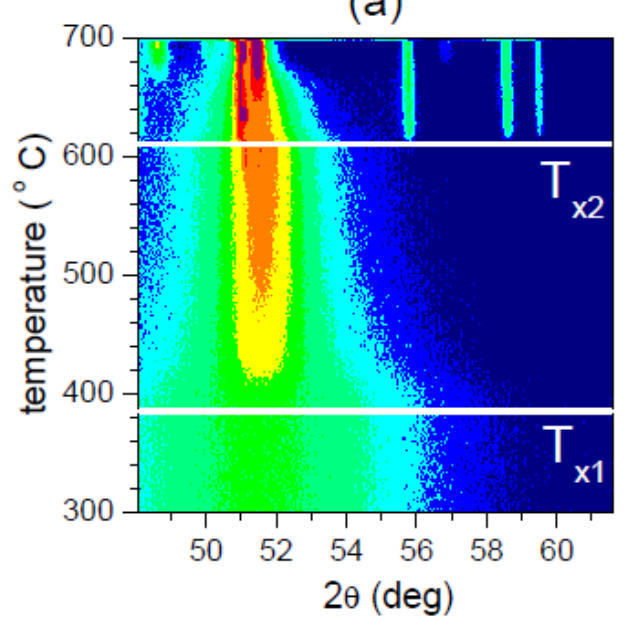

(b)

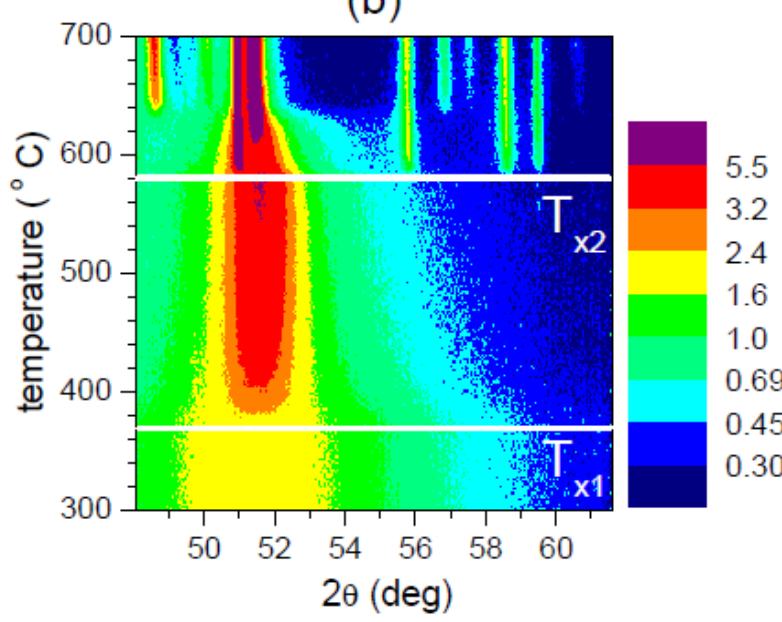

Fig. 4. Contour plots of diffractograms recorded by in situ DSR from the air (a) and the wheel (b) side of the $\left(\mathrm{Fe}_{1-x} \mathrm{Co}_{x}\right)_{76} \mathrm{Mo}_{8} \mathrm{Cu}_{1} \mathrm{~B}_{15}(x=0.5)$ alloy. Crystallization temperatures $T_{x 1}$ and $T_{x 2}$ are indicated by the horizontal white lines.

In the third temperature region where $T>T_{x 2}$, additional crystalline phases have evolved. They were identified as fcc- $\mathrm{Fe}_{23} \mathrm{~B}_{6}(x=0)$ and $\mathrm{Fe}(\mathrm{Co}, \mathrm{Mo})_{23} \mathrm{~B}_{6}(x=0.25,0.5)$. In early stages of the $2^{\text {nd }}$ crystallization, a CoMoB crystalline phase was identified in the $x=0.5$ alloy. Above the temperature of $\sim 660{ }^{\circ} \mathrm{C}$, formation of the tetrahedral $\mathrm{B}_{2} \mathrm{Mo}_{2} \mathrm{Fe}$ phase is observed for $x=0$ [11].

Using the contour plots in Figs. 2-4, the $T_{x 1}$ and $T_{x 2}$ values were determined. They are plotted as a function of the cobalt concentration in Fig. 5 . The estimated uncertainty in the determination of the crystallization temperatures is of $\pm 3^{\circ}$. The corresponding error bars in Fig. 5 are almost equal to the size of the symbols.

The onset of the first $\left(T_{x 1}\right)$ and the second $\left(T_{x 2}\right)$ crystallization processes occurs earlier at the wheel side. Temperature differences in $T_{x 1}$ between the air and the wheel side are of $13^{\circ}$, $10^{\circ}$, and $23^{\circ}$ as determined for $x=0,0.25$, and 0.5 , respectively. In the case of $T_{x 2}$, the values of $7^{\circ}, 15^{\circ}$, and $30^{\circ}$ were obtained. It is noteworthy that for the Co-containing alloys, variations in $T_{x 1}$ and $T_{x 2}$ observed for both sides scale with the Co content. Doubling the amount of Co from $x=0.25$ to $x=0.5$ has doubled also the temperature separation.

As seen in Fig. 5, both stages of crystallization start at lower temperatures in the alloys with higher Co contents. One can also compare the deviations between $T_{x 2}$ and $T_{x 1}$ for both sides of the ribbon-shaped samples. At the air side, they represent temperature separations of $177^{\circ}, 216^{\circ}$, and $217^{\circ}$ for $x=0,0.25$, and 0.5 , respectively. At the wheel side, the crystallization temperatures differ between the second and the first structural transformation 
by $183^{\circ}, 211^{\circ}$, and $210^{\circ}$, correspondingly. However, in contrary to the temperature deviations observed between both sides of the ribbon, the Co addition has practically no effect upon the temperature interval between the onsets of the second and the first crystallization stages.

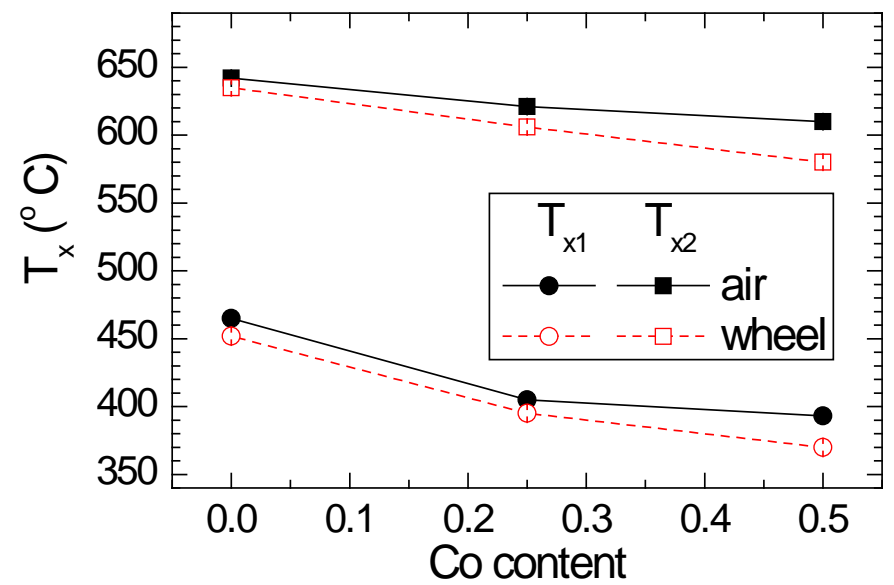

Fig. 5. Crystallization temperatures $T_{x 1}$ and $T_{x 2}$ plotted against the Co content in the $\left(\mathrm{Fe}_{1-x} \mathrm{Co}_{x}\right)_{76} \mathrm{Mo}_{8} \mathrm{Cu}_{1} \mathrm{~B}_{15}$ alloys as derived from in situ DSR taken from both air and wheel sides of the ribbons. The lines are only guides to the eye.

We have performed quantitative analysis of all data obtained from in situ DSR experiments shown in Figs. 2-4. The crystalline contents and positions of the principal reflection peaks were derived from fits of the diffractograms with Gauss and Lorentz lines. They were assigned to the amorphous phase and newly formed nanocrystals, respectively.

The areas under the narrow lines can be considered to be proportional to the relative amounts of bcc-Fe and/or bcc-(Fe,Co) nanocrystals that have evolved from original amorphous precursors during the heat treatment. They are plotted against the annealing temperature in Fig. 6. In order to avoid any influence of crystalline phases that start appearing at the onset of the second crystallization step, the analyses were terminated well below $T_{x 2}$ of the particular alloys.

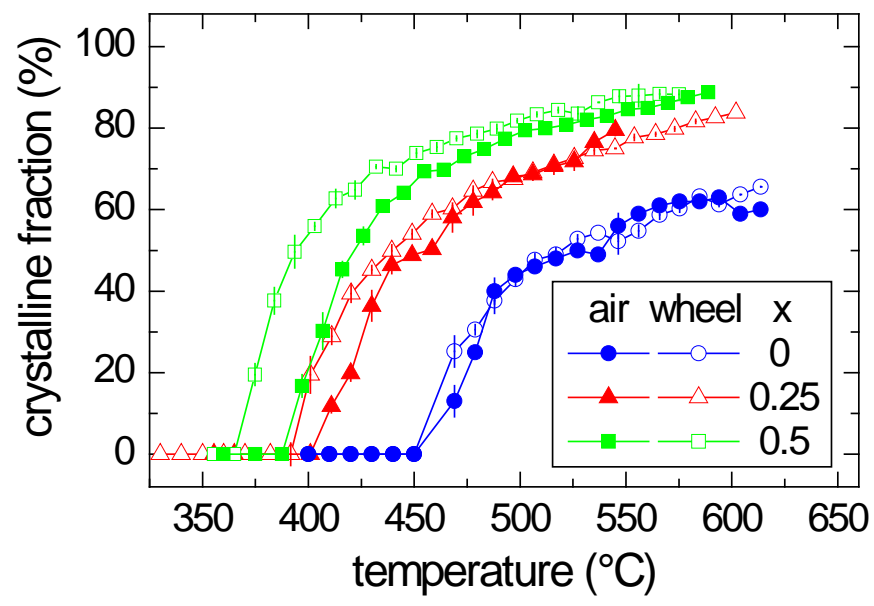

Fig. 6. Crystalline fraction plotted against the temperature of annealing as obtained from the deconvolution of in situ DSR data recorded from the air (full symbols) and the wheel (open symbols) sides of the $\left(\mathrm{Fe}_{1-x} \mathrm{Co}_{x}\right)_{76} \mathrm{Mo}_{8} \mathrm{Cu}_{1} \mathrm{~B}_{15}$ alloy. Solid lines are only guide to the eye. 
It should be noted that the data presented in Fig. 6 need not necessarily correspond to the actual contents of nanocrystals because the diffractograms were recorded only from a limited range of $2 \theta$ angles. A systematic error of about $\pm 3 \%$ can be estimated due to truncation of some parts of the diffractograms mostly in small $2 \theta$ angles region. An example of such fitting is demonstrated in Fig. 7 for the wheel side and $x=0.5$ at the temperature of $450{ }^{\circ} \mathrm{C}$. The error bars plotted in Fig. 6 were obtained from the fitting procedure. Despite the above mentioned restrictions, the overall trends in the temperature evolution of the crystalline contents are maintained. The temperatures of the onset of the first crystallization $T_{x 1}$ determined from Fig. 6 are in agreement with those presented in Fig. 5.

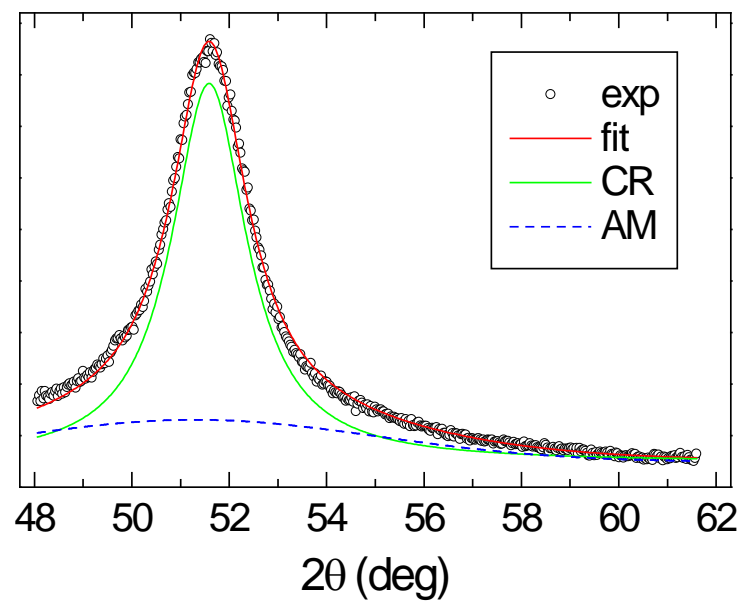

Fig. 7. Deconvolution of a diffractogram obtained from the wheel side of the $\left(\mathrm{Fe}_{1-x} \mathrm{Co}_{x}\right)_{76} \mathrm{Mo}_{8} \mathrm{Cu}_{1} \mathrm{~B}_{15}, x=0.5$ alloy at $450{ }^{\circ} \mathrm{C}$. The individual components correspond to the crystalline phase (CR) and to the residual amorphous matrix (AM).

DSC records obtained from the investigated samples are plotted in Fig. 8. The onset of crystallization is marked with arrows. Reasonable agreement between DSR and DSC data is achieved. Small deviations observed might be caused by different annealing conditions. DSC was acquired under inert atmosphere whereas DSR was performed in vacuum.

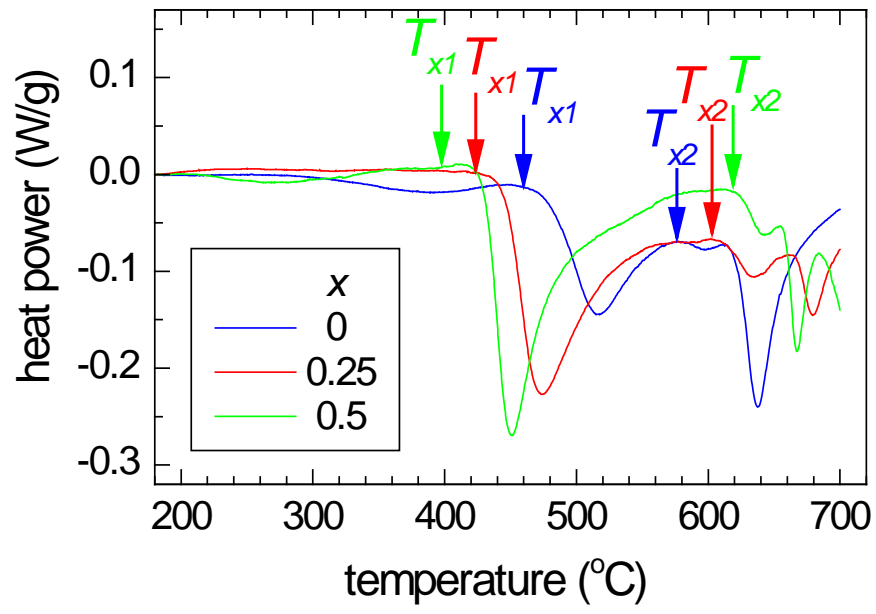

Fig. 8. Differential scanning calorimetry of the $\left(\mathrm{Fe}_{1-x} \mathrm{Co}_{x}\right)_{76} \mathrm{Mo}_{8} \mathrm{Cu}_{1} \mathrm{~B}_{15}$ alloy. Temperature of the onset of crystallization is marked with arrows. 
Positions of the reflections from DSR were used to calculate the lattice parameters of the bcc phases. They are shown in Fig. 9 as a function of temperature for all investigated samples.

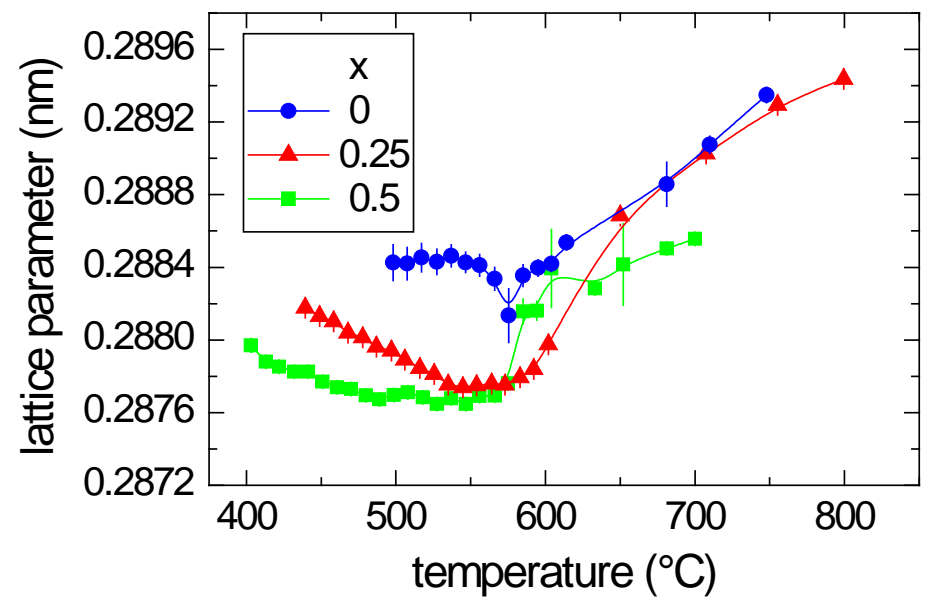

Fig. 9. Lattice parameter plotted against the annealing temperature for the $\left(\mathrm{Fe}_{1-x} \mathrm{Co}_{x}\right)_{76} \mathrm{Mo}_{8} \mathrm{Cu}_{1} \mathrm{~B}_{15}$ alloy. Solid lines are only guide to the eye.

The Co-free alloy $(x=0)$ shows rather high lattice parameters which means that probably Mo is incorporated into the bcc lattice thus causing its expansion. In the $x=0.25$ and 0.5 alloys, lower values are observed depending upon the Co content. This indicates that Co atoms apparently expel molybdenum from the bcc lattice. The initial decrease of the lattice parameter with temperature can be caused by two possible effects: (i) Mo that is incorporated into the nanograins diffuses out with increasing temperature and (ii) the lattice parameter at lower temperatures, i.e., when the grains start to evolve, is influenced by an interface effect [36]. A rapid increase of the lattice parameter indicates temperature-induced lattice expansion.

\subsection{Nuclear forward scattering of synchrotron radiation}

The use of in situ DSR described above helped in the elucidation of the temperature induced structural transformations in the studied $\left(\mathrm{Fe}_{1-\mathrm{x}} \mathrm{Co}_{\mathrm{x}}\right)_{76} \mathrm{Mo}_{8} \mathrm{Cu}_{1} \mathrm{~B}_{15} \mathrm{MG}$. Because of the applied experimental geometry (see Section 2.2.1), the reported results were obtained from subsurface regions that extend only to a limited depth $(\sim 5 \mu \mathrm{m})$. On one hand, this approach has revealed structural differences between both sides of the investigated ribbon-shaped specimens. On the other hand, information related to areas far away from the surface $(>5 \mu \mathrm{m})$ of the samples is not available. However, this can be gathered from another in situ synchrotron-based technique, namely with nuclear forward scattering (NFS) of synchrotron radiation. Some basic aspects of this less established method are briefly mentioned in Section 2.2.2. Let us emphasise that NFS scans hyperfine interactions of the ${ }^{57} \mathrm{Fe}$ resonant nuclei that are contained in the whole investigated material. As the hyperfine interactions are governed by local atomic order, information on both structural arrangement and magnetic ordering can be obtained simultaneously from NFS interferograms. The latter represent plots of the number of 'delayed' photons, which are emitted by the sample during the process of its de-excitation, as a function of time that has elapsed after the excitation with a synchrotron-radiation pulse.

In order to facilitate the NFS experiments, samples enriched in ${ }^{57} \mathrm{Fe}$ to about $50 \%$ have been used. Contour plots obtained from NFS interferograms recorded every minute during continuous temperature increase (with the ramping-rate of $10 \mathrm{~K} / \mathrm{min}$ ) are shown in Fig. 10 for 
$x=0$ and $x=0.25$ alloys. Vertical axes represent the temperature of the investigated samples while the elapsed time is given on the horizontal ones. The counts of the registered photons (intensities) are colour coded in a logarithmic scale. In total, about 90 interferograms were acquired during one experiment. Only those which exhibit pronounced development of hyperfine parameters with temperature are shown in Fig. 10.

(a)

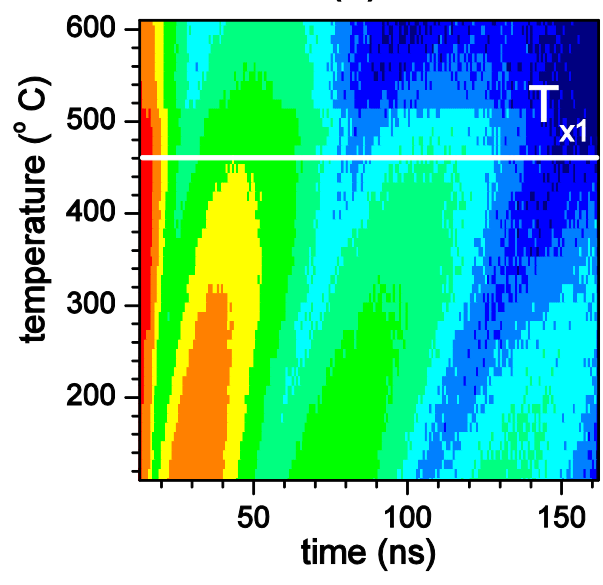

(b)

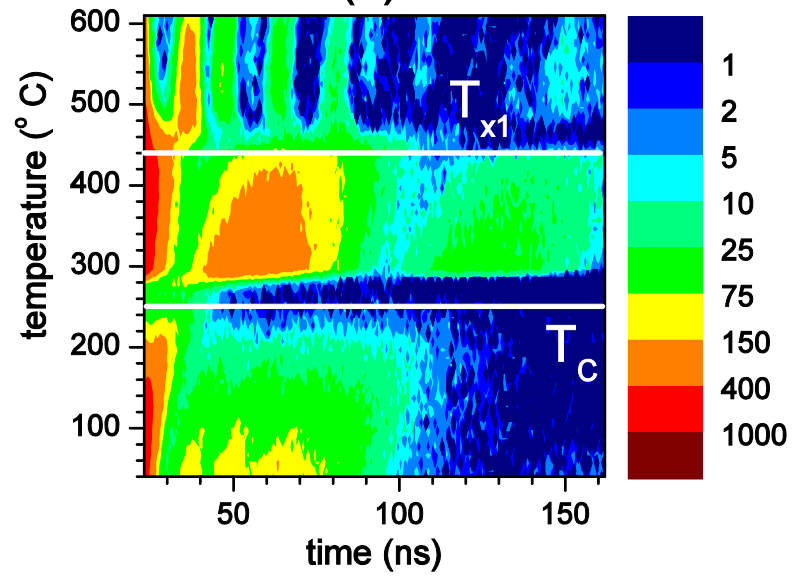

Fig. 10. Contour plot of 3-D nuclear forward scattering data of $\left(\mathrm{Fe}_{1-x} \mathrm{Co}_{x}\right)_{76} \mathrm{Mo}_{8} \mathrm{Cu}_{1} \mathrm{~B}_{15}$ for $x=0$ (a) and $x=0.25$ (b). Crystallization $T_{x 1}$ and Curie $T_{C}$ temperatures are indicated by the horizontal white lines.

Clear distinctions are seen in the character of the contour plots for $x=0$ and $x=0.25$. The as-quenched Co-free $\mathrm{Fe}_{76} \mathrm{Mo}_{8} \mathrm{Cu}_{1} \mathrm{~B}_{15} \mathrm{MG}$ shows close-to-room Curie temperature $T_{C}=40{ }^{\circ} \mathrm{C}$ [37]. That is why the obtained NFS records in Fig. 10a correspond to quadrupole electric interactions that are typical for paramagnetic materials. Consequently, the presented NFS interferograms can be described by the quantum beats that stem from distributed quadrupolar doublets. Their evolution with increasing annealing temperature does not show any qualitative changes. Even the onset of the first crystallization $T_{x 1}$ is quite difficult to unveil only from these data. This is partly also due to the fact that the produced bcc-Fe nanograins are small ( $\sim 5-8 \mathrm{~nm})$ and not very abundant (see Section 3.1). Consequently, the corresponding hyperfine interactions at $T>T_{x 1}$ are considerably smeared out and their contribution to the NFS time records is hindered with the signal from the residual amorphous matrix. Because of these unfortunate difficulties, the $x=0$ alloy is obviously an inappropriate candidate to demonstrate the diagnostic potential of the in situ NFS technique. On the other hand, the $x=0.5$ sample exhibits stronger magnetic interactions [28] which give rise to higher $T_{C}$. At the same time, this composition features lower temperature of the first crystallization $T_{x 1}$ (Fig. 8) than the $x=0.25$ alloy. Consequently, one can expect narrower temperature region between $T_{C}$ and $T_{x 1}$ in which pure paramagnetic amorphous phase exists and the evolution of NFS time records would be rather rapid. We have also considered technological limitations that are associated with the production of iron enriched alloys. That is why the main attention is paid merely to the results achieved from the $x=0.25$ alloy in the following part.

The character of NFS interferograms in Fig. 10b changes abruptly at two distinct temperatures, viz. $T_{C}$ and $T_{x 1}$. They subdivide the inspected temperature interval into three diverse regions. In the first one where $T<T_{C}$, the studied alloy is completely amorphous and it undergoes the second order structural transition from ferromagnetic to paramagnetic state at $T_{C}$. Addition of cobalt to the original NANOPERM-type Fe-Mo-Cu-B alloy modifies its 
chemical composition. In this way, a HITPERM-type Fe,Co-Mo-Cu-B MG is produced. The latter is ferromagnetic at room temperature in the as-quenched amorphous state.

Evaluation of interferograms in this region was accomplished according to the physical model consisting of two distributions of hyperfine magnetic fields. They were assigned to short-range order (SRO) regions with high $(\sim 22 \mathrm{~T})$ and low $(\sim 8 \mathrm{~T})$ average hyperfine magnetic fields in ambient conditions (room temperature). They originate from deviations in chemical composition around the resonant iron atoms (chemical SRO) as well as from the influence of topological SRO.

In the intermediate temperature region where $T_{C}<T<T_{x 1}$, the qualitative behaviour of NFS interferograms is similar to that observed in the $x=0$ alloy. From a structural point of view, the sample is still amorphous. It is, however, already paramagnetic and that is why only relatively simple quantum beats of electric quadrupole interactions are observed. Consequently, the experimental data corresponding to this temperature interval were modelled with one distribution of quadrupole splitting.

Selected examples of interferograms from these two temperature regions are shown in Fig. 11a. After moderate temperature increase to $107^{\circ} \mathrm{C}$, the sample exhibits amorphous structure with quantum beats assigned to hyperfine magnetic interactions. They are demonstrated by small periodic oscillations in the $35-65$ ns time region. At $167{ }^{\circ} \mathrm{C}$, minor traces of these beats are still visible but they vanish completely at $T_{C} \sim 247{ }^{\circ} \mathrm{C}$. Beyond this temperature (e.g., at $307^{\circ} \mathrm{C}$ ), the interferograms demonstrate quantum beats that correspond to electric quadrupole hyperfine interactions. Here, the sample is still fully amorphous but already paramagnetic. This character of the quantum beats persists, from a qualitative point of view, till the onset of crystallization (see for example the interferogram at $427^{\circ} \mathrm{C}$ in Fig. $11 \mathrm{~b}$ ).

(a)

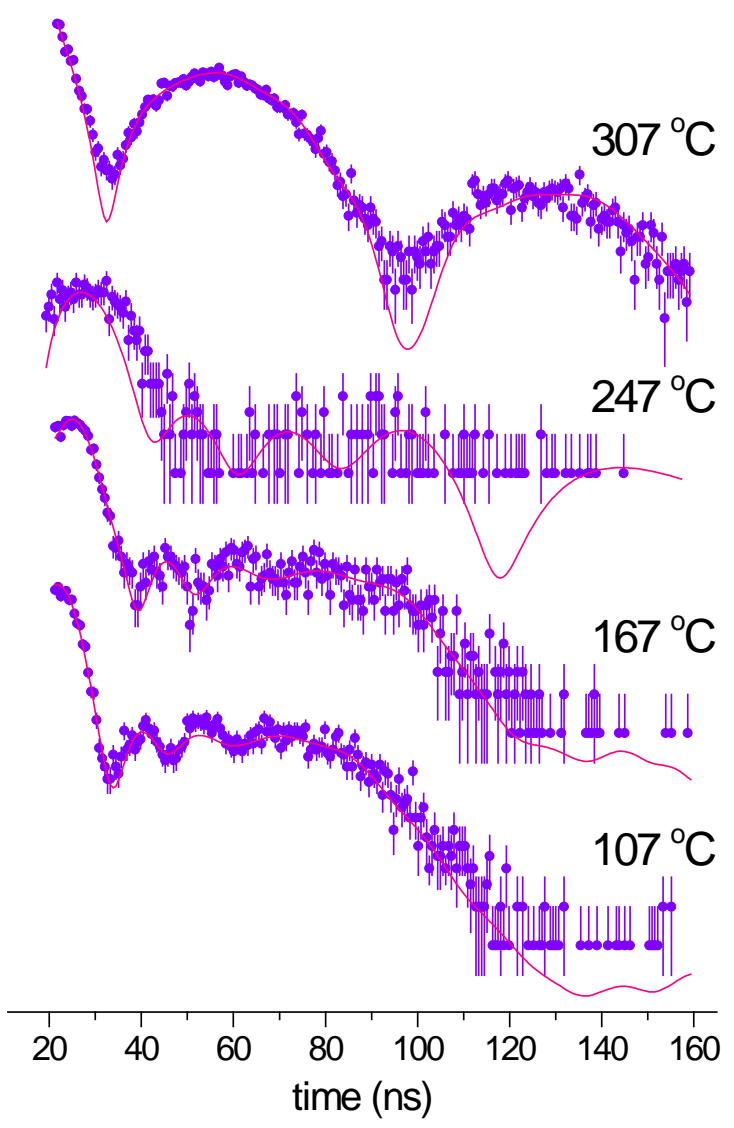

(b)

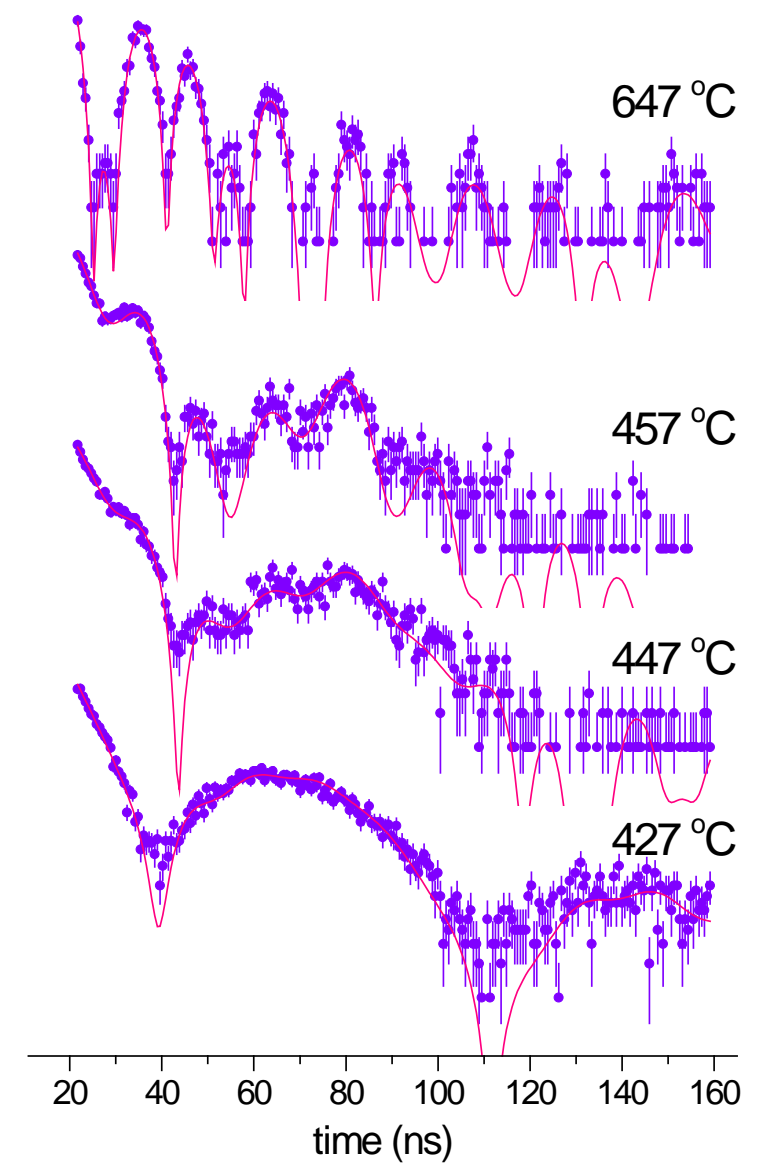


Fig. 11. Selected records obtained from NFS for the $\left(\mathrm{Fe}_{1-x} \mathrm{Co}_{x}\right)_{76} \mathrm{Mo}_{8} \mathrm{Cu}_{1} \mathrm{~B}_{15}(x=0.25)$ at the indicated temperatures in the vicinity of $T_{C}$ (a) and $T_{x 1}$ (b). Experimental data (symbols) are fitted (solid lines) according to the model described in the text.

The third temperature region $T>T_{x 1}$ is characterized by continuous formation of nanocrystalline bcc-(Fe,Co) grains. They are identified by the corresponding quantum beats that appear in the time region $43-83 \mathrm{~ns}$, and represent hyperfine magnetic fields. Figure 11b shows, however, an interferogram at $447^{\circ} \mathrm{C}$ in which the magnetic quantum beats are better visible. With increasing annealing temperature these rather narrow magnetic quantum beats gradually quickly evolve as the corresponding nanocrystalline phase does. This is demonstrated by an interferogram taken at $457^{\circ} \mathrm{C}$. Finally, at the end of the heating process at $\sim 647^{\circ} \mathrm{C}$ the sample is already well crystallized and the resulting interferogram clearly shows magnetic structure.

Evaluation of the interferograms in this temperature region was accomplished with a model that took into consideration both the presence of a residual amorphous matrix that was, however, in paramagnetic state as well as the newly formed bcc-(Fe,Co) nanocrystals. The former was fitted with one distribution of quadrupole splitting that was applied actually already in the $T>T_{C}$ region. The latter were represented by four components with hyperfine magnetic fields (not distributed) whose relative fractions were derived from a binomial distribution of the Co nearest neighbours:

$$
P(n)=\frac{8 !}{n ! \cdot(8-n) !} \cdot x^{n} \cdot(1-x)^{(8-n)}
$$

where $n$ is the number of Co nearest neighbours and $x$ is the Co concentration $(x=0.25)$. The number of sextets used was determined by a condition of a minimum contribution $P(n)>5 \%$ that could be unambiguously distinguished in the interferograms. Consequently, $0,1,2$, and 3 Co nearest neighbours in the bcc-Fe,Co lattice were considered. In order to ensure a convergent fitting procedure, the relative fractions of the magnetic components were fixed to the values obtained from Equ. (1). Their hyperfine magnetic fields were fitted without any restrictions. We have tried also more magnetic components, however the fit became unstable.

As it can be seen from Fig. 11, the obtained fits (solid lines) satisfactorily represent the measured experimental data (full symbols). Some deviations are observed at the transition temperatures $\left(T_{C}, T_{x 1}\right)$ where the applied physical models change. This is well visible at $T_{C}$ because of an abrupt transformation of the character of the hyperfine interactions from dipole magnetic into quadrupolar electric ones. In addition, the beat intensities are dramatically reduced for times higher than 60 ns due to the broad distribution of the hyperfine magnetic field. This is a nice demonstration of the second order phase transformation observed in situ that alters the magnetic microstructure while the structural arrangement is maintained.

The above mentioned physical models were used to fit all the measured interferograms. It should be noted that during acquisition of the NFS data, temperature was continuously increasing with the ramping-rate of $10 \mathrm{~K} / \mathrm{min}$. Evolution of the content of bcc-(Fe,Co) nanocrystalline phase and the residual amorphous matrix is plotted in Fig. 12 against the annealing temperature. The onset of crystallization is determined at $T_{x 1} \sim 435{ }^{\circ} \mathrm{C}$, which is slightly higher than those determined from DSR for the air and the wheel sides of the ribbonshaped sample. This is due to the fact that the crystallization starts first on the surfaces and then progresses into the bulk that is scanned by NFS.

The evolution of relative fractions of the crystalline phase can be used to describe the kinetics of crystallization. Using isothermal annealing experiments, we have introduced 
relatively simple model of the evolution of nanograins [29] and satisfactory match between the experimental and theoretical data was achieved. Because one deals in this case with dynamical temperature regimes, more elaborated crystallization models should be employed that describe non-isothermal time evolution [38, 39]. We would like to emphasis that the main aim of this study is to show how synchrotron-based techniques of DSR and NFS can be used in the description of structural transformations during dynamic annealing. The application of different kinetics models is beyond the scope of this study.

One more important point should be mentioned, however. While DSR experiments provide information on the total amount of bcc nanocrystalline grains, NFS can go even further. By the help of the fitting models described above, it is possible to distinguish among structurally different iron occupational sites within the bcc lattice. Through hyperfine interactions (viz. hyperfine magnetic fields) acting upon the ${ }^{57} \mathrm{Fe}$ resonant nuclei, presence of varying number of their cobalt nearest neighbours is evidenced. Temperature development of relative contributions of iron sites with none (Co0), one (Co1), two (Co2), and three (Co3) cobalt nearest neighbours are plotted in Fig. 12, too. The sum of these four components constitutes the overall contents of nanograins (CR).

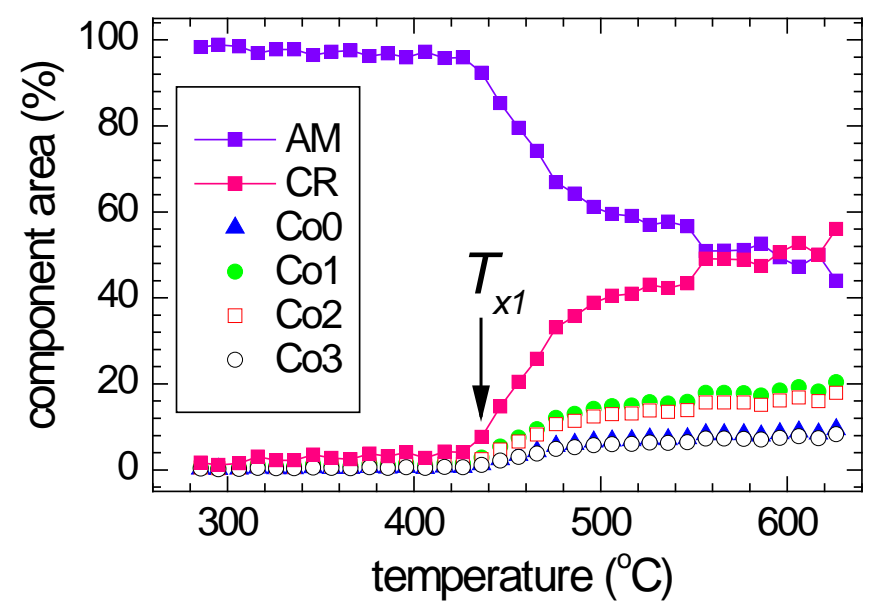

Fig. 12. Fraction of the residual amorphous matrix (AM) and bcc-(Fe,Co) crystalline phase (CR) plotted against the annealing temperature as obtained from the fitting of in situ NFS data of the $\left(\mathrm{Fe}_{1-x} \mathrm{Co}_{x}\right)_{76} \mathrm{Mo}_{8} \mathrm{Cu}_{1} \mathrm{~B}_{15}(x=0.25)$ alloy. Fractions of crystalline sites with different amounts of Co nearest neighbours ( $\mathrm{CoO}-\mathrm{Co} 3)$ are also given. Solid lines are only guide to the eye. The arrow indicates the onset of crystallization $T_{x 1}$.

Average hyperfine magnetic fields of the amorphous and nanocrystalline phases are displayed in Fig. 13. In the temperature region $T<T_{C}$ in Fig. 13a, the fields represent different SRO arrangements of the resonant ${ }^{57} \mathrm{Fe}$ nuclei in the amorphous structure. The highfield component ( $22 \mathrm{~T}$ at close-to-room temperature) represents Fe neighbours that are surrounded predominantly with Co while the low-field one $(\sim 8 \mathrm{~T})$ reflects contributions from other constituent elements (Mo, B). Both average magnetic hyperfine fields are decreasing with temperature and they are supposed to vanish at $T_{C}$. It should be noted that the values of 3-5 $\mathrm{T}$ for the low-field component in the vicinity of $T_{C}$ are comparable in strength with the newly developing electric quadrupole interactions and can be hardly distinguished one from another. In this respect, small hyperfine magnetic fields can be treated as quadrupole splitting. On the other hand, the high-field component that attains $\sim 11 \mathrm{~T}$ at $T_{C}$ contributes only marginally at higher temperatures.

Here, a huge diagnostic potential of the NFS technique which scans simultaneously both the local structural arrangement and hyperfine interactions is nicely seen. Though the overall 
structure is amorphous, contributions from regions with different chemical SRO and their evolution can be followed in situ as a function of increasing temperature of measurement. Such data can be hardly obtained by another technique. Majority of conventional analytical tools treat amorphous structures as structurally isotropic regions which usually provide only a featureless (broadened and/or halloed) signal.

Hyperfine magnetic fields in Fig. 13b belong to four narrow components that were used to reconstruct the contributions of the bcc-(Fe,Co) nanocrystals. Shortly after the onset of crystallization they show growing trend with temperature. This is due to progressing formation of the bcc lattice that is still building-up. Similar effect is observed by DSR for the lattice parameter in Fig. 9. Eventually, the hyperfine fields arrived at their average values of $\sim 27.2 \mathrm{~T}, \sim 29.2 \mathrm{~T}, \sim 30.5 \mathrm{~T}$, and $\sim 31.6 \mathrm{~T}$ and follow the expected temperature dependences. According to the obtained hyperfine magnetic field values, the particular components were assigned to iron atom sites with zero, one, two, and three cobalt nearest neighbours in the bcc(Fe,Co) crystalline lattice.

(a)

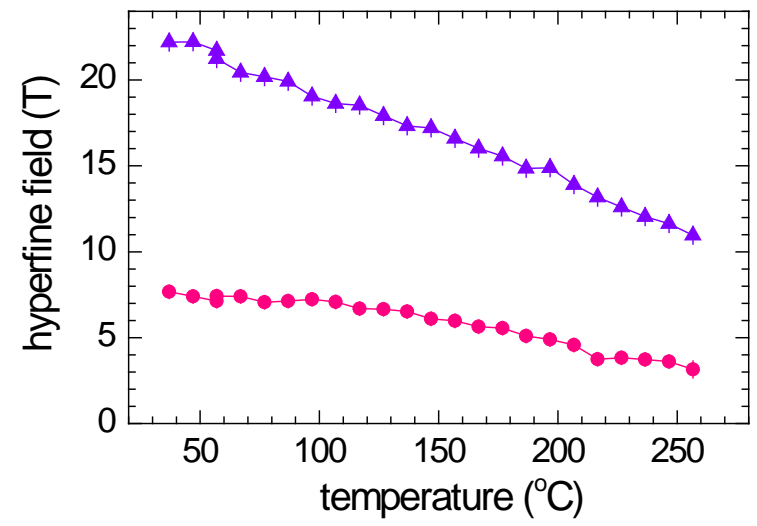

(b)

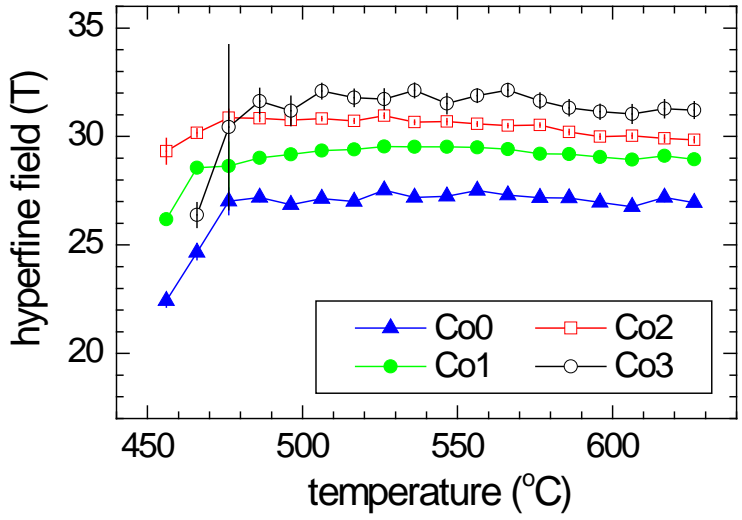

Fig. 13. Hyperfine magnetic fields of the fitted components in amorphous state (a) and of individual crystalline lattice sites with zero (Co0), one (Co1), two (Co2), and three (Co3) cobalt nearest neighbours (b) plotted against the annealing temperature as obtained from the fitting of in situ NFS data of the $\left(\mathrm{Fe}_{1-x} \mathrm{Co}_{x}\right){ }_{76} \mathrm{Moo}_{8} \mathrm{Cu}_{1} \mathrm{~B}_{15}(x=0.25)$ alloy. Solid lines are only guide to the eye.

Similarly to the case of the $T<T_{C}$ region, where the system is fully amorphous, the in situ NFS technique is very exceptional also here. The mutual relationship between structural arrangement and the corresponding hyperfine interactions enables the formation of the bccFe,Co lattice to be indirectly followed making use of its hyperfine magnetic fields. The practical applicability of the method rests with the fact that even particular crystalline lattice sites, which have eventually different numbers of Co nearest neighbours, can be identified and, subsequently, their behaviour with continuously varying temperature can be also independently and separately studied.

As mentioned above, in the intermediate temperature region where $T_{C}<T<T_{x 1}$, the experimental data were modelled with one distribution of quadrupole splitting. After the onset of crystallization $\left(T>T_{x 1}\right)$, the amorphous residual matrix still persists and taking into consideration relatively high temperatures of the NFS experiments it was reconstructed also by one distribution of quadrupole splitting $(Q S)$. The obtained average $Q S$ values are plotted against the temperature of annealing in Fig. 14.

Quadrupole splitting is a measure of charge density at the nuclei. The quantitative change in $Q S$ values at $\sim 450{ }^{\circ} \mathrm{C}$ coincides with the onset of crystallization. In the temperature 
range $\mathrm{T}>500{ }^{\circ} \mathrm{C}$ where the crystalline phase is already quite well developed, stabilization of QS occurs. It should be noted that the physical model that we have used to describe the behaviour of the studied system comprises distributions of hyperfine parameters (viz., amorphous residual matrix) and well defined values of the crystalline components. Consequently, the fitting procedure is very complex. From this perspective we should admit that the absolute values of QS could be debatable.

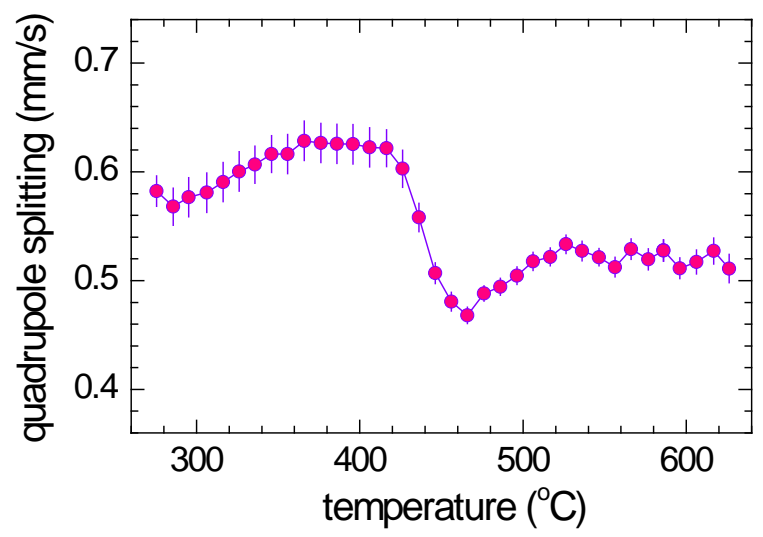

Fig. 14. Average values of quadrupole splitting of the amorphous residual matrix plotted against the annealing temperature as obtained from the fitting of in situ NFS data of the $\left(\mathrm{Fe}_{1-x} \mathrm{Co}_{x}\right)_{76} \mathrm{Mo}_{8} \mathrm{Cu}_{1} \mathrm{~B}_{15}(x=0.25)$ alloy. Solid line is only guide to the eye.

This situation is quite similar to that which the researchers were faced with about 30 years ago when the first attempts to refine broad Mössbauer spectra of amorphous metallic glasses have appeared. In order to ensure consistency of the obtained hyperfine parameters we have checked their temperature evolution rather than to relay upon fitting of a single NFS interferogram. This approach is closely discussed in [8] for the case of conventional Mössbauer spectra. As demonstrated by Fig. 13 and Fig. 14, we hope that we have succeeded in obtaining relevant temperature dependences of the particular hyperfine parameters. To our best knowledge, similar data does not exist in a literature and we have introduced the obtained hyperfine parameters (namely QS) in order to provoke discussion on the ways how to fit NFS data of amorphous and/or nanocrystalline alloys. Nevertheless, the methodological aspects of NFS data evaluation are beyond the scope of the current work.

Here, we wanted to point at the particular features of the NFS technique which allows in situ transformation studies aimed at understanding evolution of specific materials properties during these transient periods. The latter can be followed through the evolution of hyperfine parameters. More examples of such studies can be found in our most recent papers [40, 41].

\section{Conclusions}

The formation of nanocrystalline structure is decisive for macroscopic physical properties that in turn govern the practical applications of these materials. Under external conditions of prolonged elevated temperature, compositional dependent structural transformations may occur. The influence of temperature was followed during in situ experiments using the techniques based upon synchrotron radiation.

Effects of cobalt substitution on kinetics of crystallization was investigated for $\left(\mathrm{Fe}_{1-\mathrm{x}} \mathrm{Co}_{\mathrm{x}}\right)_{76} \mathrm{Mo}_{8} \mathrm{Cu}_{1} \mathrm{~B}_{15}(x=0,0.25,0.5)$ nanocrystalline alloy by in situ diffraction of synchrotron radiation. We observed a tendency of the first and the second crystallization temperature to decrease with raising the cobalt content. It was shown that the surface 
crystallization starts earlier at the wheel side than at the air side of ribbon-shaped samples. Structural characteristics comprising temperature evolution of relative amount of newly formed crystalline phases as well as their lattice parameters were followed in situ during heat treatment of the original metallic glass. These parameters were obtained from analyses of the diffractograms taken during short time intervals.

Along with structural characteristics, also magnetic states of the studied system were inspected via temperature development of the associated hyperfine interactions. The latter were derived from time interferograms that were recorded by nuclear forward scattering of synchrotron radiation. This technique enables separate evaluation of the contributions that stem from structurally different regions within the investigated samples including the newly formed nanocrystals and the residual amorphous matrix. In addition, their magnetic microstructures can be also assessed. We have shown that even minor Co content $(x=0.25)$ has a substantial effect not only upon the magnetic behaviour of the alloy but also upon its structure. Making use of hyperfine magnetic fields it was possible to unveil structurally diverse positions of $\mathrm{Fe}$ atoms that reside in a nanocrystalline lattice with different number of Co nearest neighbours.

Extremely high brilliance of the present sources of synchrotron radiation makes doable experiments that are capable of providing information on-fly during continuous variation of external conditions (e.g., temperature). Such approach is hardly affordable with other sources of radiation. In this way, the use of synchrotron-based techniques paves a completely new road to the understanding of technologically important details on the formation of nanocrystalline structures by heat treatment of the originally amorphous precursors.

\section{Acknowledgement}

The authors gratefully acknowledge the support by the project LO1305 of the Ministry of Education, Youth and Sports of the Czech Republic and the projects, CZ.1.07/2.3.00/20.0155, and CZ.1.07/2.3.00/30.0041. Allocation of synchrotron beamtimes at Helmholtz-Zentrum Berlin and European Synchrotron Radiation Facility is also gratefully acknowledged.

\section{References}

[1] M. E. McHenry and D. E. Laughlin, Acta Mater., 2000, 48, 223.

[2] Y. Yoshizawa, A. Oguma and K. Yamauchi, J. Appl. Phys., 1988, 64, 6044.

[3] K. Suzuki, N. Kataoka, A. Inoue, A. Makino and T. Masumoto, Mater. Trans. JIM, 1990, 31, 743.

[4] M. A. Willard, D. E. Laughlin, M. E. McHenry, D. Thoma, K. Sickafus, J. O. Cross and V. G. Harris, J. Appl. Phys., 1998, 84, 6773.

[5] A. Hernando, J. Phys.: Condens. Matter., 1999, 11, 9455.

[6] G. Herzer, Acta Mater., 2013, 61, 718.

[7] S. Stankov, B. Sepiol, T. Kaňuch, D. Scherjau, R. Würschum and M. Miglierini, J. Phys.: Condens. Matter., 2005, 17, 3183.

[8] M. Miglierini and J.-M. Grenèche, J. Phys.: Condens. Matter., 2003, 15, 5637.

[9] M. Miglierini, M. Kopcewicz, B. Idzikowski, Z. E. Horváth, A. Grabias, A. I. Škorvánek, P. Dluzewski and Cs. C. Daróczi, J. Appl. Phys., 1999, 85, 1014.

[10] M. Pavuk, M. Miglierini, M. Vujtek, M. Mashlan, R. Zboril and Y. Jiraskova, J. Phys.: Condens. Matter., 2007, 19, 216219.

[11] M. Paluga, P. Švec, D. Janičkovič, D. Muller, P. Mrafko and M. Miglierini, Rev. Adv. Mater. Sci., 2008, 18, 481.

[12] M. Miglierini, A. Lančok and J. Kohout, J., Appl. Phys. Lett., 2010, 96, 211902. 
[13] U. Köster, U. Schünemann, M. Blank-Bewersdorff, S. Brauer, M. Sutton and G. B. Stephenson, Mater. Sci. Eng. A, 1991, 133, 611.

[14] J. M. Borrego, C. F. Conde, M. Millán, A. Conde, M. J. Capitán and J. L. Joulaud, Nanostructured materials, 1998, 10, 575.

[15] C. R. M. Afonso, C. Bolfarini, W. J. Botta Filho and C. S. Kiminamim, J. NonCrystalline Solids, 2006, 352, 3404.

[16] J. Bednarčík, R. Nicula, M. Stir and E. Burkel, J. Magn. Magn. Mat., 2007, 316, 823.

[17] A. R. Yavari, A. Le Moulec, A. Inoue, N. Nishiyama, N. Lupu, E. Matsubara, W. J. Botta, G. Vaughan, M. Di Michiel and Å Kvick, Acta Mater., 2005, 53, 1611.

[18] H. F. Poulsen, J. A. Wert, J. Neuefeind, V. Honkimäki and M. Daymond, Nat. Mater., 2005, 4, 33.

[19] S. Mechler, G. Schumacher, I. Zizak, M. P. Macht and N. Wanderka, Appl. Phys. Lett., 2007, 91, 021907.

[20] N. Mattern, M. Stoica, G. Vaughan and J. Eckert, Acta Mater., 2012, 60, 517.

[21] G. Wang, N. Mattern, J. Bednarčík, R. Li, B. Zhang and J. Eckert, Acta Mater., 2012, 60, 3074.

[22] S. Michalik, J. Michalikova, M. Pavlovic, P. Sovak, H.-P. Liermann and M. Miglierini, Acta Mater., 2014, 80, 309.

[23] M. Miglierini, T. Kaňuch, M. Pavúk, P. Švec, G. Schumacher, I. Zizak and Y. Jirásková, Hyperfine Int., 2008, 183, 31.

[24] C. F. Conde, A. Conde, D. Janičkovič and P. Švec, J. Magn. Magn. Mater., 2006, 304, e739.

[25] G. Vlasák, C. F. Conde, D. Janičkovič and P. Švec, Mater. Sci. Eng. A, 2007, 449-451, 464.

[26] C. F. Conde, J. M. Borrego, J. S. Blázquez, A. Conde, P. Švec and D. Janičkovič, J. Alloys Compounds, 2011, 5091994.

[27] T. Kaňuch, M. Miglierini, A. Lančok, P. Švec and E. Illeková, Acta Phys. Pol., 2008, 113, 63.

[28] M. Miglierini, J. Phys.: Conf. Series, 2010, 217, 012092.

[29] M. Miglierini, V. Procházka, S. Stankov, P. Svec Sr., M. Zajac, J. Kohout, A. Lancok, D. Janickovic and P. Svec, Phys. Rev. B, 2013, 86, 020202(R).

[30] K. Suzuki, A. Makito, N. Kataika, A. Inoue and T. Masumoto, Mater. Trans. JIM., 1991, 32, 93.

[31] G. Mülhaupt and R. Rüffer, Hyperfine Int., 1999, 123/124, 13.

[32] G. V. Smirnov, Hyperfine Int., 1999, 123/124, 31.

[33] R. Rüffer, C.R. Physique, 2008, 9, 595.

[34] W. Sturhahn and E. Gerdau, Phys Rev B, 1994, 49, 9285.

[35] W. Sturhahn, Hyperfine Interact., 2000, 125, 149.

[36] M. Dittrich and G. Schumacher, Mater. Sci. Eng. A, 2014, 604, 27.

[37] M. Hasiak, M. Miglierini, J. Kaleta and M. Bujdoš, Acta. Phys. Pol. A, 2015, 127,608.

[38] F. Liu, F. Sommer and E. J. Mittemeijer, J. Mater. Sci., 2004, 39, 1621.

[39] J. S. Blázquez, J. M. Borrego, C. F. Conde, A. Conde and S. Lozano-Pérez, J. Alloys Compounds, 2012, 544, 73.

[40] M. Miglierini, V. Procházka, R. Rüffer and R. Zbořil, Acta Mater., 2015, 91, 50.

[41] V. Procházka, V. Vrba, D. Smrčka, R. Rüffer, P. Matúš, M. Mašláň and M. B. Miglierini, J. Alloys Compounds, 2015, 638, 398. 